\title{
Parameters Estimation of Interharmonic Based on State Space Model
}

\author{
Yuan Shiji, Pei Bin, Liu Zhihua, Huang Wenjing, and Sun Mingfeng \\ Luoyang Electronic Equipment Test Center, Jiyuan, Henan 459000, China \\ Correspondence should be addressed to Yuan Shiji; yuanke520@sohu.com
}

Received 21 May 2013; Revised 6 November 2013; Accepted 7 November 2013; Published 13 February 2014

Academic Editor: Grigori M. Sisoev

Copyright (C) 2014 Yuan Shiji et al. This is an open access article distributed under the Creative Commons Attribution License, which permits unrestricted use, distribution, and reproduction in any medium, provided the original work is properly cited.

\begin{abstract}
The special Hankel matrix is structured from interharmonic sampling, which is described by state space model. A method of parameters estimation based on state space model is proposed, which can achieve interharmonics frequency, amplitude and, phase of the joint estimation. The simulation results show that the method can effectively restrain white Gaussian noise, with superior performance.
\end{abstract}

\section{Introduction}

Traditional power system harmonic analysis method based on Fourier transform (FFT) has the frequency spectrum revelation and the stockade effect, so there are limitations in the detection of harmonic $[1,2]$. Some methods have been applied to estimate the damped exponential model parameters based on modern signal processing theory, such as Pisarenko harmonic decomposition, AR model, and multisignal classification (MUSIC) [3-5]. They have high estimation accuracy, robustness, and other advantages, but most of these methods that need to search in the frequency domain spectra did not realize the frequency, amplitude, and other parameters of the joint estimation.

The parameter estimation method based on state space model (SSM) can inhibit the channel between any zero-mean noise, and it also improves the resolution of parameter estimation [6-9]. The special Hankel matrix is structured from interharmonic sampling; then a method of parameter estimation based on state space model has achieved the joint estimation. The simulation results show that the method can effectively restrain Gaussian color noise, with superior performance.

\section{Interharmonic Signal Model}

The interharmonics are defined as follows in IEC-61000-2-2: there is not an integer multiple signal between the frequency and the fundamental frequency in the voltage and current signal harmonic. Power system harmonics and inter-harmonic detection signal can be expressed as [3].

Consider the following:

$$
\begin{aligned}
y(n) & =s(n)+v(n) \\
& =\sum_{i=1}^{P} \alpha_{i} \cos \left(2 \pi f_{i} n+\varphi_{i}\right)+v(n),
\end{aligned}
$$

where $y(n)$ is sampling signal; $n$ is sampling points; $P$ is interharmonic number; $\alpha_{i}$ is the $i$ th inter-harmonic component of amplitude; $f_{i}$ is the $i$ th inter-harmonic frequency; $\varphi_{i}$ is the initial angle; $v(n)$ is a zero-mean white Gaussian noise.

\section{Parameters Estimation Algorithm Based on SSM}

Equation (1) is transformed for complex frequency signal, because the state space model is transformed from the ARMA model [10]. With the Hilbert transform (1) can be expressed as follows:

$$
y(m)=\sum_{i=1}^{P} \alpha_{i} \exp \left(j 2 \pi f_{i} m+\varphi_{i}\right)+v(n) .
$$


Equation (2) $y(m)$ can be described using SSM [11, 12]:

$$
\begin{gathered}
X(m+1)=F X(m)+g \delta(m), \\
y(m)=h X(m)+J \delta(m),
\end{gathered}
$$

where $y(m)$ stands for the output of the fourth order mixed cumulants of $y(n), X(m)$ stands for the state variables, $\delta(m)$ stands for the pulse function, $F$ stands for the system state matrix, and $g, h$, and $J$ stand for constant matrix. The initial conditions of $X(0)=0 . y(m)$ can be obtained form the state space theory $[11,12]$ :

$$
y(m)= \begin{cases}J & m=0 \\ g F^{m-1} g & m>0 .\end{cases}
$$

Data matrix $Y$ is constructed as follows:

$$
Y=\left(\begin{array}{ccc}
y(1) & \cdots & y(L) \\
\vdots & \ddots & \vdots \\
y(N-L+1) & \cdots & y(N)
\end{array}\right),
$$

where the $L$ stands for the correlation window length $(2 N / 3) N$ stands for the data length. Another expression of $Y$ can be obtained with substitution of (4) into (5):

$$
Y=\left[\begin{array}{c}
h \\
h F \\
h F^{2} \\
\vdots \\
h F^{N-L}
\end{array}\right] \cdot\left[g, F g, F^{2} g, \ldots, F^{L-1} g\right]=\Omega \cdot \Lambda
$$

The $\Omega$ and $\Lambda$ are defined as follows:

$$
\Omega=\left[\begin{array}{c}
h \\
h F \\
h F^{2} \\
\vdots \\
h F^{N-L}
\end{array}\right] ; \quad \Lambda=\left[g, F g, F^{2} g, \ldots, F^{L-1} g\right] .
$$

The $\Omega$ and $\Lambda$ have the following properties:

$$
\Omega_{1}=\Omega_{2} F ; \quad \Lambda_{1}=F \Lambda_{2},
$$

where the subscripts 1 and 2 represent deletion of the first row of (column) and the last row (column) of corresponding matrix. Singular value decomposition of matrix $Y$ is

$$
Y=\left[\begin{array}{ll}
U_{\mathrm{sn}} & U_{n}
\end{array}\right]\left(\begin{array}{cc}
\sum_{\mathrm{sn}} & 0 \\
0 & \sum_{n}
\end{array}\right)\left(\begin{array}{c}
V_{\mathrm{sn}}^{*} \\
V_{n}^{*}
\end{array}\right),
$$

where the subscripts sn and $n$ stand for the signal and noise subspace and “*” stands for conjugate transpose. The left standard matrix $\left[U_{\text {sn }} U_{n}\right]$ is composed by the signal matrix $U_{\text {sn }}$ and noise matrix $U_{n}$, and the right standard matrix $\left[\begin{array}{ll}V_{s n} & V_{n}\end{array}\right]$ is composed by the signal matrix $V_{\text {sn }}$ and noise matrix $V_{n} . \sum_{\text {sn }}$ is the diagonal matrix of singular values corresponding to the signal, and $\sum_{n}$ is the diagonal matrix of singular values corresponding to the noise. The matrix $Y$ should be removed from the noise caused by the singular value and its corresponding eigenvector in order to increase the accuracy of estimated state matrix $F$; that is, $\widetilde{Y}=U_{\mathrm{sn}} \sum_{\mathrm{sn}} V_{\mathrm{sn}}^{*}$. The $P$-order approximate estimation of $\Omega$ and $\Lambda$ can be obtained from (9) with $P$ as the model order. The state transfer matrix approximation can be obtained according to (8).

Consider the following:

$$
\widehat{F}=\left(\Omega_{2}{ }^{*} \Omega_{2}\right)^{-1} \Omega_{2}{ }^{*} \Omega_{1} .
$$

The “*” represents the conjugate transpose. Let eigenvalues of matrix $\widehat{F}$ be $\lambda_{1}, \lambda_{2}, \ldots, \lambda_{p}$ :

$$
\lambda\{\widehat{F}\}=\left\{\lambda_{1}, \lambda_{2}, \ldots, \lambda_{p}\right\}
$$

Define a new matrix $\Omega_{N}$ as follows:

$$
\Omega_{N}=\left[\begin{array}{c}
h \\
h F \\
h F^{2} \\
\vdots \\
h F^{N}
\end{array}\right]
$$

Then $\Omega_{N} g=y^{T}, g=\left(\Omega_{N}^{*} \Omega_{N}\right)^{-1} \Omega_{N}^{*} y^{T} \cdot \lambda_{i}$ is the state space corresponding to the transfer function of the pole from the spectral estimation theory.

Consider the following:

$$
\varphi_{i}=\ln \left|\lambda_{i}\right| ; \quad f_{i}=\frac{-\phi_{i}}{2 \pi},
$$

where $\lambda_{i}=\left|\lambda_{i}\right| \angle \phi_{i}$. There is $F m_{i}=\lambda_{i} m_{i}$, and $m_{i}$ is eigenvectors of $\lambda_{i}$. The matrix $M$ is composed of $m_{i}$,

$$
M=\left[\begin{array}{llll}
m_{1} & m_{2} & \cdots & m_{p}
\end{array}\right] ;
$$

that is, $F M=M Y$,

$$
\Upsilon=\operatorname{diag}\left[\begin{array}{llll}
\lambda_{1} & \lambda_{2} & \cdots & \lambda_{p}
\end{array}\right], \quad F=M \Upsilon M^{-1} .
$$

The substitution into (4) yields $y(n)=h M Y^{n-1} M^{-1} g=$ $h M M^{-1} g \Upsilon^{n-1}$.

Make the following:

$$
M^{-1}=\left[\begin{array}{c}
v_{1} \\
v_{2} \\
\vdots \\
v_{p}
\end{array}\right] \text {, }
$$

$y(n)=\sum_{i=1}^{p}\left(h m_{i}\right)\left(v_{i} g\right) \lambda_{i}^{n-1} ; n=1, \ldots, N$. The $y(n)$ can be described in (2):

$$
\alpha_{i}=\frac{\left(h m_{i}\right)\left(v_{i} g\right)}{\lambda_{i}} .
$$

The algorithm steps of estimate model pole based on SSM method will be described as follows:

(i) constructing data matrix $Y$ according to (5);

(ii) singular value decomposition with data matrix $Y$; 


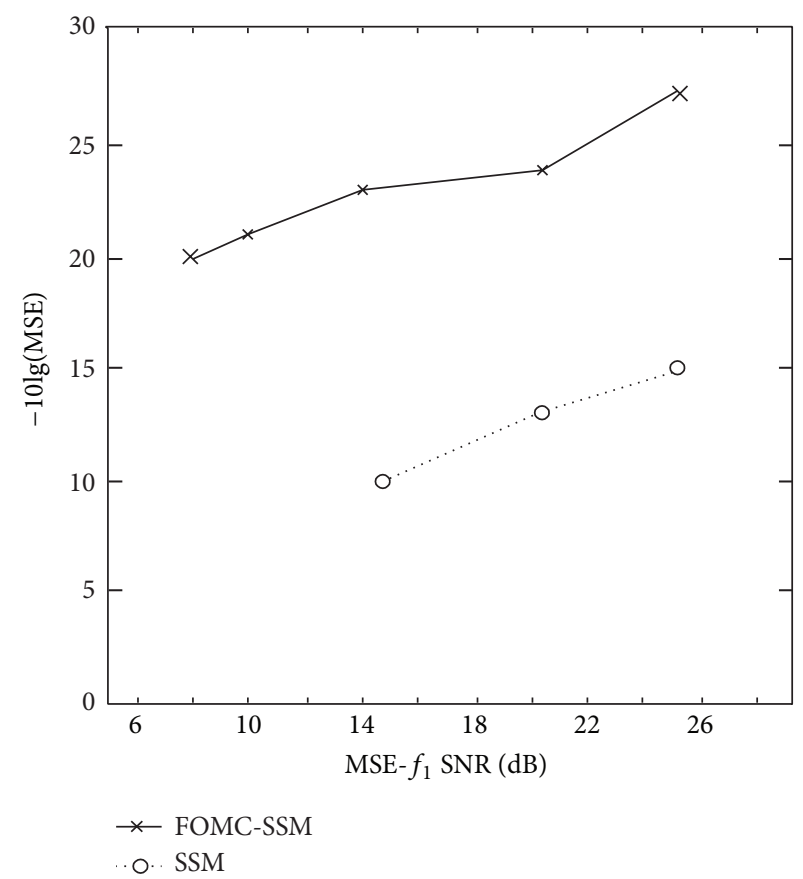

(a)

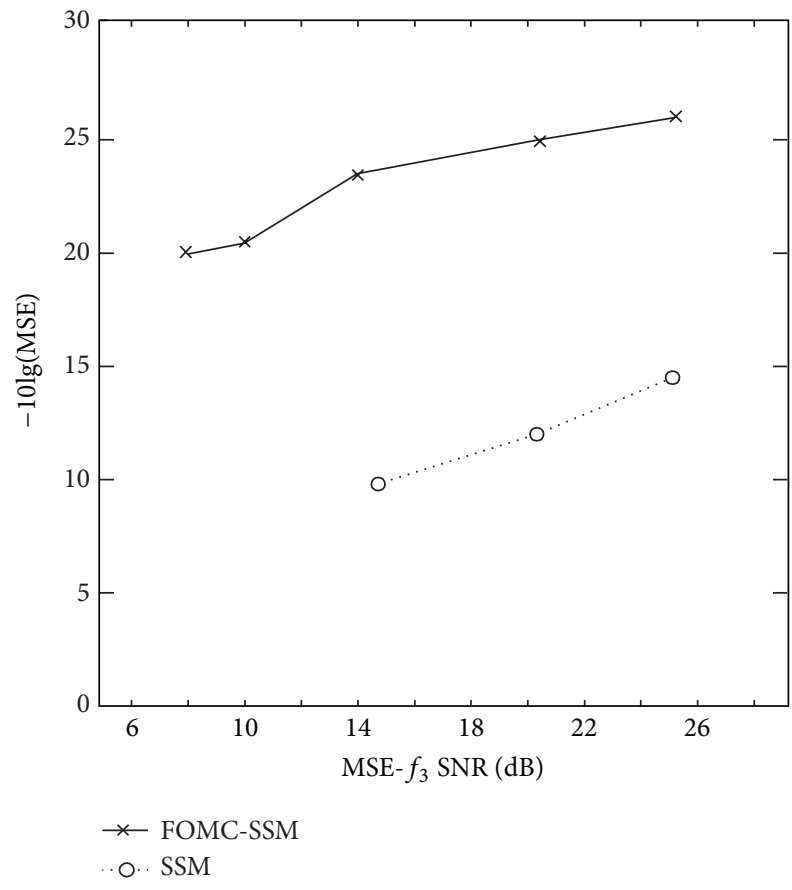

(c)

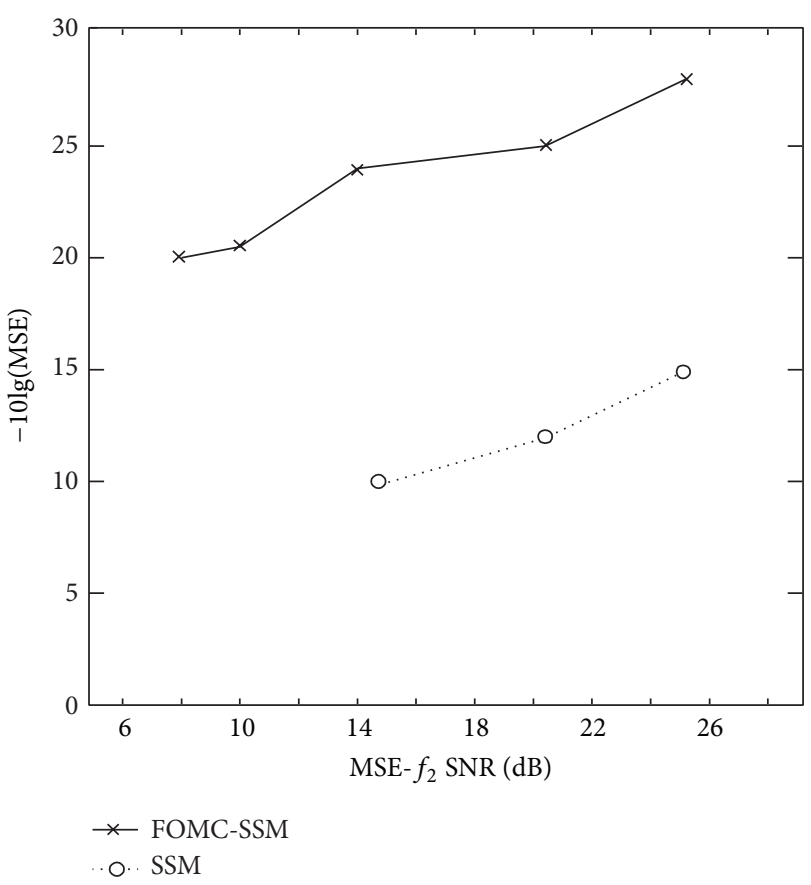

(b)

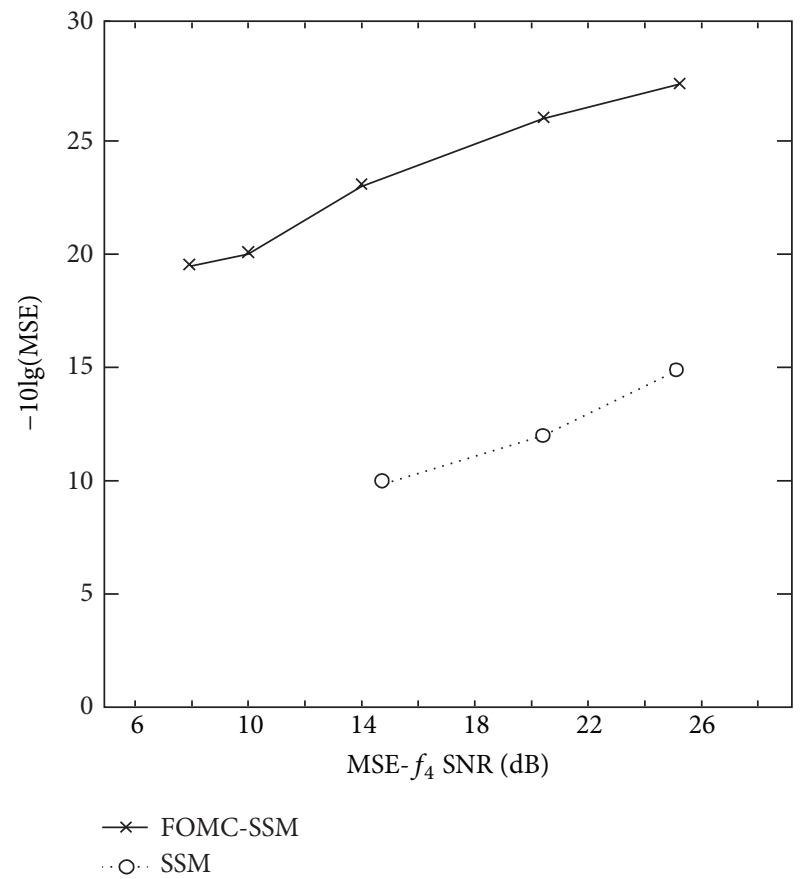

(d)

FIGURE 1: Logarithm mean square error at different method and SNR.

(iii) calculating the model order $P$ according to the literature $[11]$ and $P$-order approximation;

(iv) estimation of $\Omega$ being obtained from (9);

(v) calculating approximation of $F$ according to (10);

(vi) calculating the eigenvalues of the matrix $F$ that is the damped exponential model of pole parameters; (vii) calculating inter-harmonic component of amplitude according to (17).

\section{Simulation}

The experiment sampling signal that is inter-harmonic is described in (1), and the number of inter-harmonics is $P=3$; 


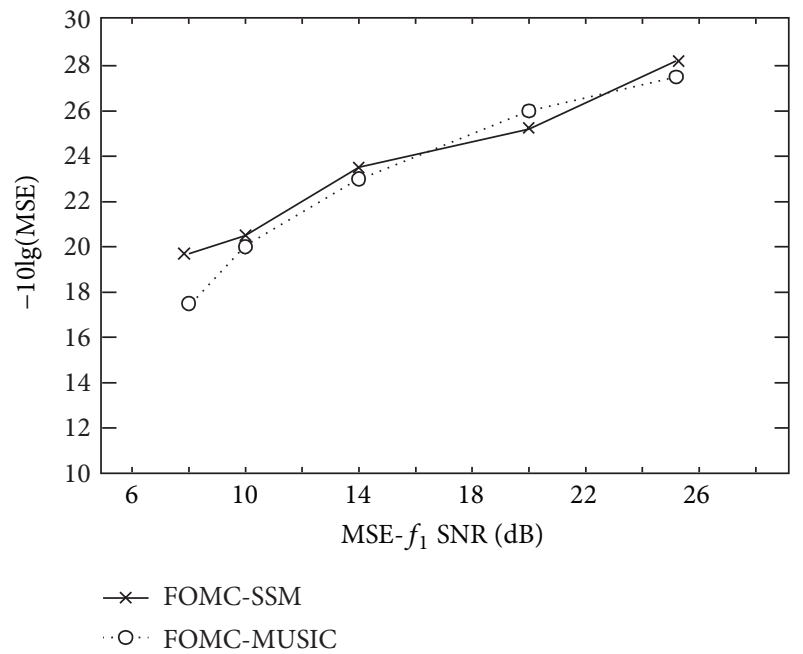

(a)

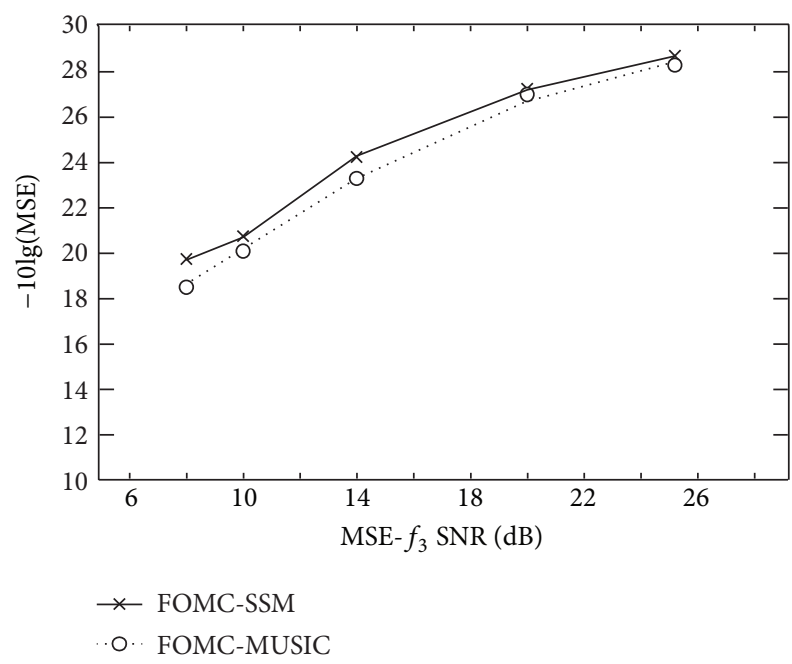

(c)

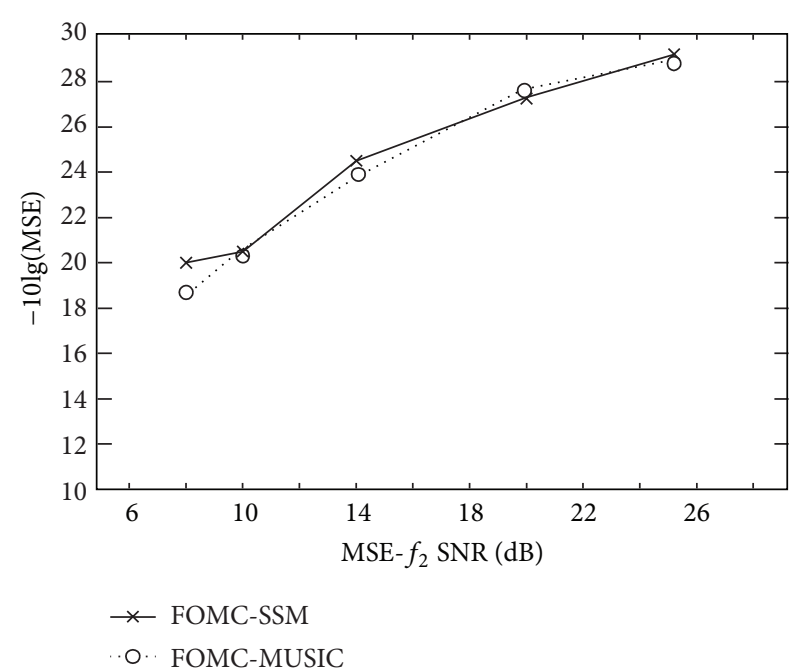

(b)

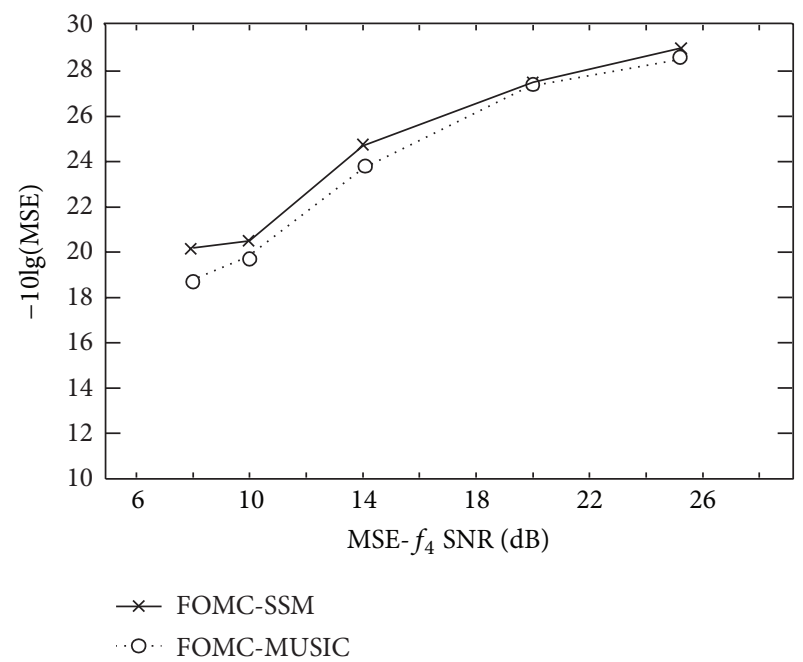

(d)

FIGURE 2: MSE of estimation frequency at different method and SNR.

sampling points are $N=128$. The parameters are set in Table 1 . Experiments with additive white Gaussian noise with $\sigma^{2}$ variance. The SNR is defined as the signal and the noise ratio of average power:

$$
\mathrm{SNR}=10 \log \left[\left(\frac{\sum_{n=1}^{N}|y(n)|^{2}}{\left(N \sigma^{2}\right)}\right)\right] .
$$

Data correlation length $L=(2 N / 3)$ and $M=(N-L-1) / 2$.

The results of this experiment are shown in Table 2 for 200 Monte-Carlo simulations at different SNR on damped exponential model parameter estimates. The method can be used to more accurately achieve damped frequency, damped factor of the joint estimation, and the method is less sensitive to the white Gaussian noise. The accuracy of damped frequency is higher than damped factor, because other parameters are estimated by the damped frequency that is firstly estimated. From this table it can also be seen that the damped frequency
TABLE 1: Interharmonic parameters.

\begin{tabular}{lccc}
\hline$i$ & 1 & 2 & 3 \\
\hline$A_{i}$ & 0.1 & 0.2 & 0.3 \\
$f_{i}$ & 0.2 & 0.4 & 0.8 \\
$\varphi_{i}$ & $\pi / 3(1.0472)$ & $\pi / 4(0.7854)$ & $\pi / 5(0.6283)$ \\
\hline
\end{tabular}

estimation is superior as the damped frequency is larger, because the signal model is closer to smooth signal with slowly damping.

The comparison of logarithm mean square error (MSE) of estimation frequency at different SNR between these methods with the traditional SSM algorithm is shown in Figure 1. The traditional SSM parameter estimation method is more sensitive to the Gaussian colored noise, while the SSM parameter estimation method based on FOMC can inhibit the Gaussian colored noise from Figure 1. 
TABle 2: White Gaussian noise, 200 simulations, and interharmonics parameter estimation bias.

\begin{tabular}{lccccccccc}
\hline $\mathrm{SNR} / \mathrm{dB}$ & $f_{1_{(0.20)}}$ & $f_{2_{(0.40)}}$ & $f_{3_{(0.80)}}$ & $\alpha_{1_{(0.10)}}$ & $\alpha_{2_{(0.20)}}$ & $\alpha_{3_{(0.30)}}$ & $\varphi_{1}(\pi / 3)$ & $\varphi_{2}(\pi / 4)$ & $\varphi_{3}(\pi / 5)$ \\
\hline 8 & $-8.9 e 2$ & $6.6 e 2$ & $3.7 e 3$ & $-3.9 e 2$ & $2.9 e 2$ & $2.7 e 2$ & $9.7 e 2$ & $7.8 e 2$ & $-5.7 e 2$ \\
10 & $-6.7 e 2$ & $-2.2 e 2$ & $-6.8 e 3$ & $2.1 e 2$ & $-1.3 e 2$ & $-1.3 e 2$ & $-6.3 e 2$ & $4.5 e 2$ & $-3.3 e 2$ \\
15 & $4 e 3$ & $1.2 e 3$ & $4.7 e 4$ & $-6.2 e 3$ & $5.1 e 3$ & $4 e 3$ & $8.7 e 3$ & $-7.9 e 3$ & $5.6 e 3$ \\
20 & $-2.1 e 4$ & $4.1 e 5$ & $2.8 e 5$ & $-7.1 e 3$ & $8.2 e 3$ & $-5.4 e 4$ & $-5.4 e 3$ & $-3.7 e 3$ & $3 e 4$ \\
$\mathbf{2 5}$ & - & - & - & $4.1 e 3$ & $-6.7 e 3$ & $-3 e 4$ & $-6.3 e 3$ & $4 e 3$ & $-6.9 e 4$ \\
\hline
\end{tabular}

Figure 2 shows that the comparison of logarithm mean square error of estimation frequency at different SNR between the methods with FOC-MUSIC algorithm is proposed in the literature [3]. The performance of this method is superior to FOC-MUSIC algorithm proposed in the literature [3] at low SNR with the same superior performance between the two estimates at high SNR from Figure 2. At the same time, this method can achieve damped frequency, damped factor of the joint estimation with less calculation.

\section{Conclusion}

Traditional inter-harmonic detection method needs to search for peaks in the frequency domain that did not realize the frequency, amplitude, and other parameters of the joint estimation. Parameter estimation method based on SSM achieves damped frequency, damped factor of the joint estimation, which avoids the search in the frequency domain spectra with superior performance.

\section{Conflict of Interests}

The authors declare that there is no conflict of interests regarding the publication of this paper.

\section{References}

[1] W. C. Zhao, W. M. Ma, and A. Hu, "FFT algorithm with high accurary for harmonic analysis in the electric machine," in Proceedings of the CSEE, vol. 21, pp. 83-87, 2001.

[2] C. J. Qi and X. H. Wang, "Inter harmonics estimation based on interpolation FFT algorithm," Transactions of China Electro Technical Society, vol. 18, no. 1, pp. 92-95, 2003.

[3] J. W. Zhang, N. C. Zhou, F. Yang, and C. X. Cuo, "Research on the inte-harmonics measure using fourth-order cumulant based multiple signal classification method," Relay, vol. 36, no. 7, pp. 19-23, 2008.

[4] S. Rouquette and M. Najim, "Estimation of frequencies and damping factors by two-dimensional ESPRIT type methods," IEEE Transactions on Signal Processing, vol. 49, no. 1, pp. 237$245,2001$.

[5] P. Chevalier, A. Ferréol, and L. Albera, "High-resolution direction finding from higher order statistics: the 2q-MUSIC algorithm," IEEE Transactions on Signal Processing, vol. 54, no. 8, pp. 2986-2997, 2006.

[6] Z.-J. Liu, X.-T. Kang, L.-L. Zhang, and Y.-W. Shi, "Tls-Hankel method based on the state space model of harmonic retrieval," Journal of Jilin University (Engineering and Technology Edition), vol. 38, no. 1, pp. 224-228, 2008.
[7] J. E. Piou, "System realization using 2-D output measurements," in Proceedings of the IEEE American Control Conference (AAC '04), pp. 2840-2845, July 2004.

[8] K. Naishadham and J. E. Piou, "A robust state space model for the characterization of extended returns in radar target signatures," IEEE Transactions on Antennas and Propagation, vol. 56, no. 6, pp. 1742-1751, 2008.

[9] S. J. Yuan, Research on Feature Extraction of Midcourse Ballistic Target Based on Wideband Information, NUDT.HU Nan, Changsha, China, 2009.

[10] K. Naishadham and J. E. Piou, "A robust state space model for the characterization of extended returns in radar target signatures," IEEE Transactions on Antennas and Propagation, vol. 56, no. 6, pp. 1742-1751, 2008.

[11] D. Z. Zhang, Linear System Theory, Tsinghua University Press, 2002.

[12] S.-J. Yuan, X.-Z. Gao, G.-H. Jin, and H.-Q. Wang, "Parameter estimation of radar target 1-D scattering centers based on SSM," Systems Engineering and Electronics, vol. 33, no. 4, pp. 779-782, 2011. 


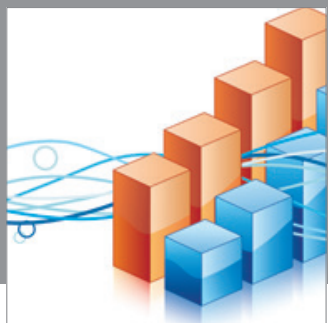

Advances in

Operations Research

mansans

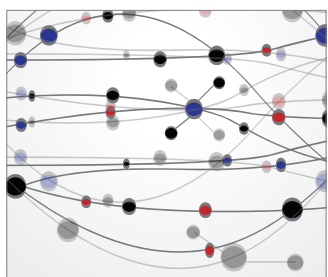

The Scientific World Journal
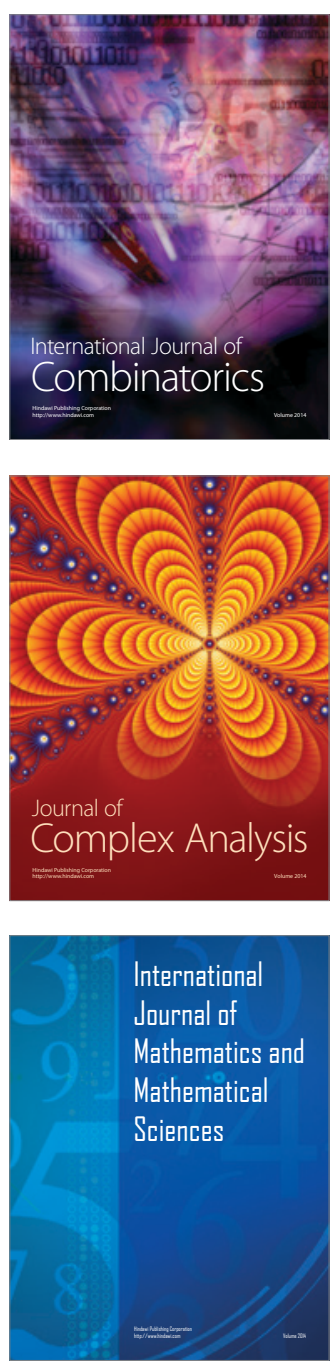
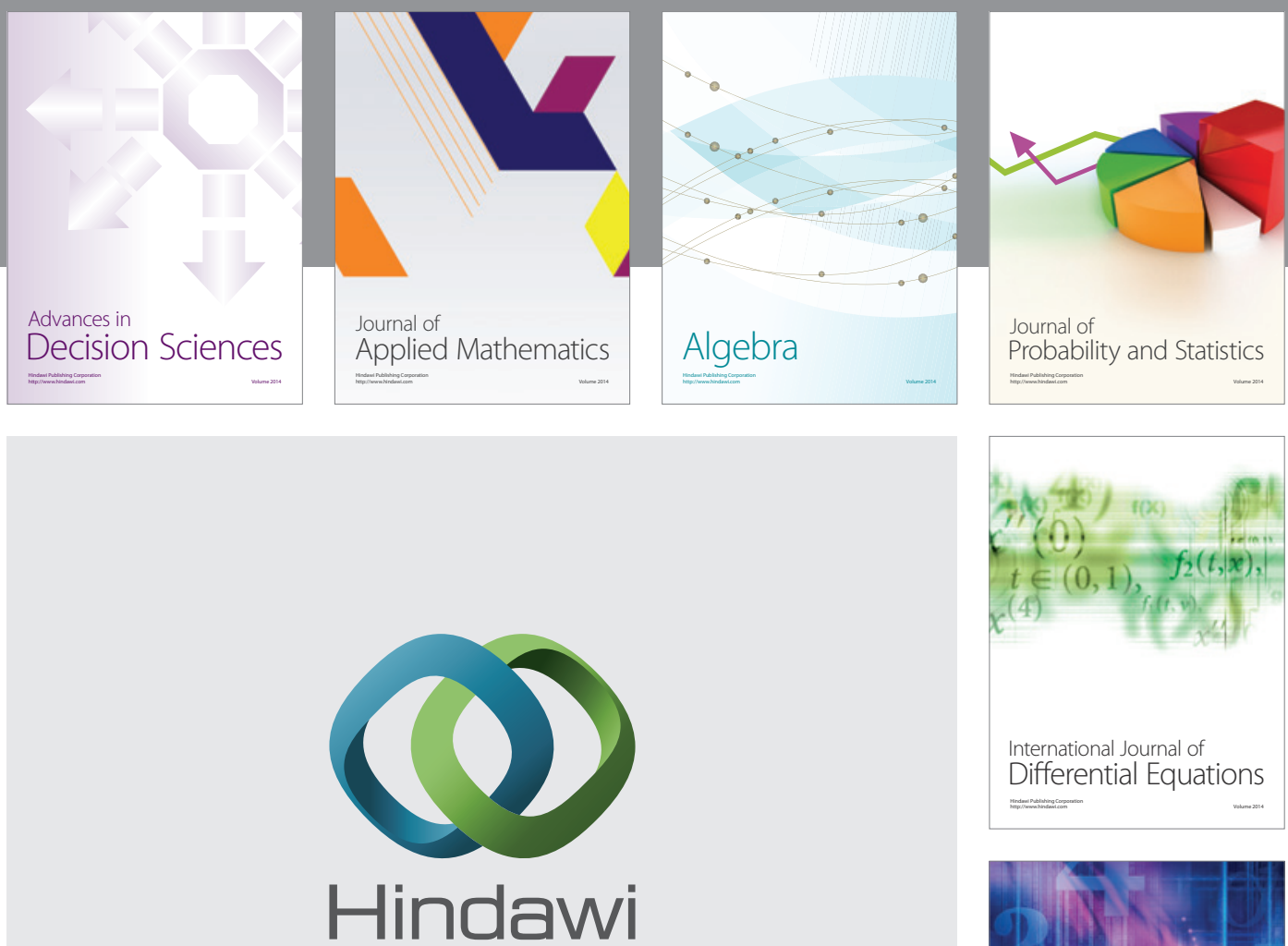

Submit your manuscripts at http://www.hindawi.com
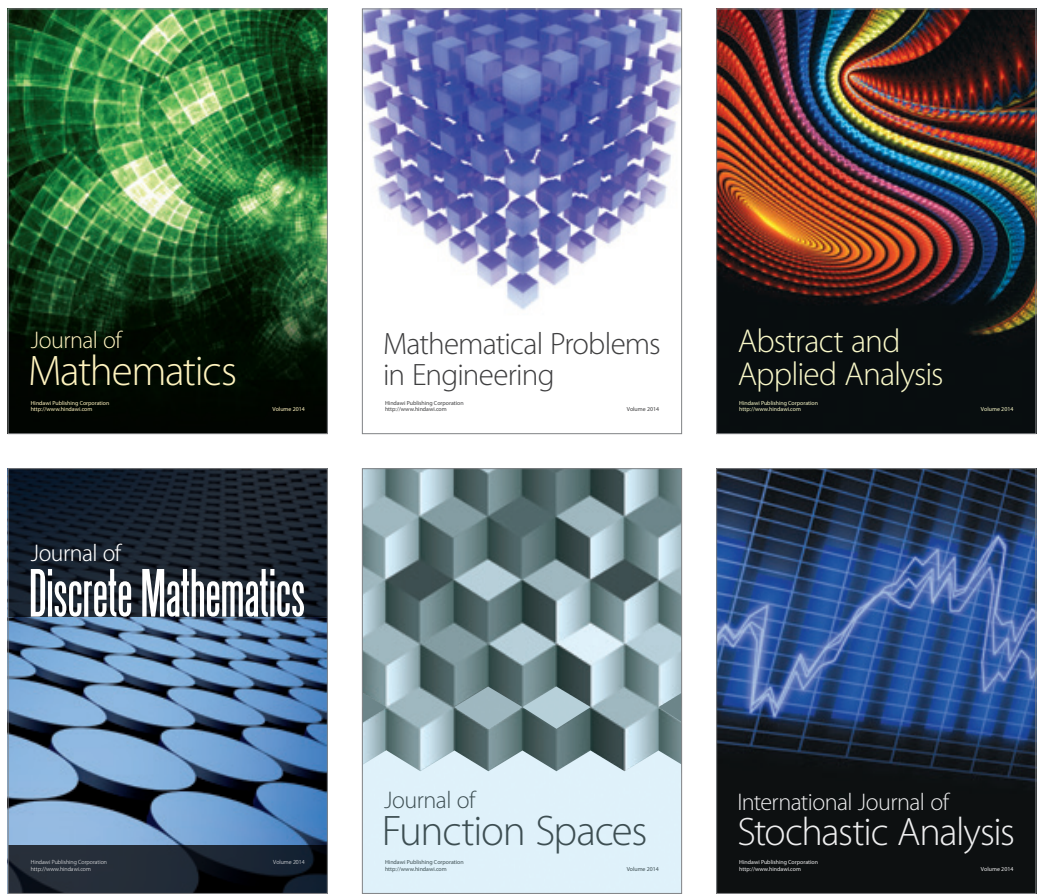

Journal of

Function Spaces

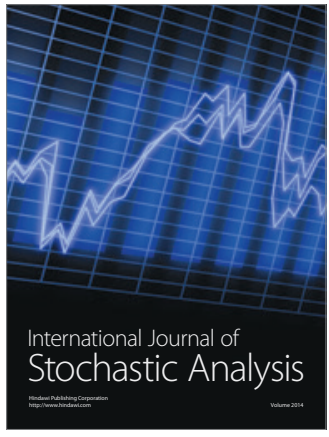

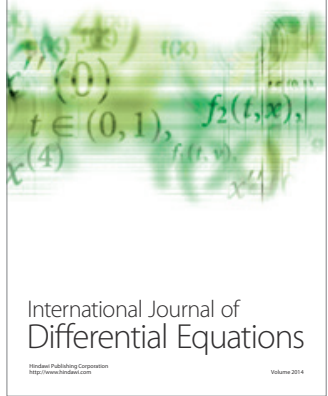
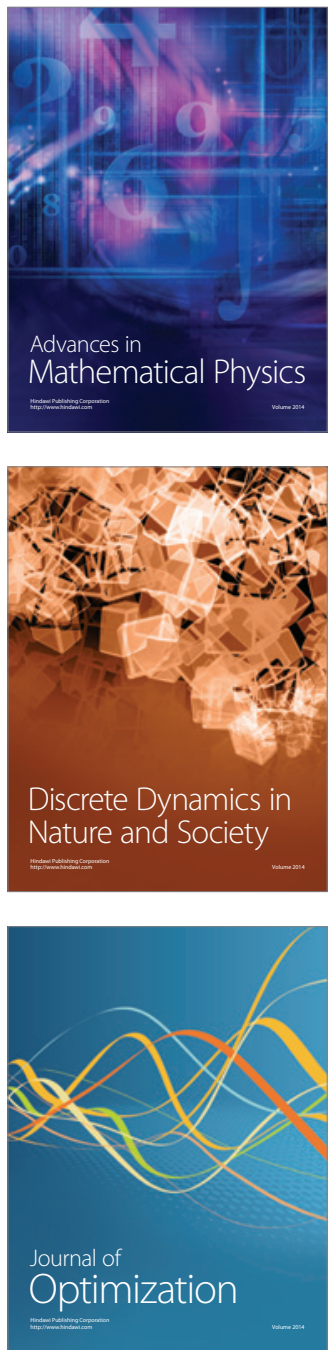